\title{
Paradoxical cerebral emboli of hypernephroma metastatic to the right ventricle five years after primary tumor resection
}

\author{
Eric Dumont, MD, ${ }^{a}$ Norman Racine, MD, ${ }^{b}$ Patricia Ugolini, MD, ${ }^{c}$ Michel Carrier, MD, ${ }^{a}$ Michel Pellerin, $M D,{ }^{a}$ and \\ Louis P. Perrault, MD, PhD, ${ }^{\text {a }}$ Montreal, Quebec, Canada
}

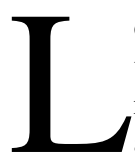
ocal extension of renal cell carcinoma via the inferior vena cava to the cardiac chambers has been previously reported. ${ }^{1}$ In most cases, the right chambers are involved and the mass is resected with the aid of cardiopulmonary bypass and circulatory arrest. ${ }^{2}$ Emboli to the lungs have been described, but to our knowledge paradoxical embolism to the brain through a patent atrial septal defect has never been reported.

\section{Clinical Summary}

A 75-year-old man had radical left nephrectomy for renal cell carcinoma with no local invasion or distant metastasis in 1994. Follow-up was uneventful. He had difficulty with speech of 5 minutes' duration associated with paresis of the left upper limb in February 2000. This was the second such episode with spontaneous resolution. The patient's history disclosed no other symptoms. Results of the physical examination were normal. The electrocardiogram revealed isolated ventricular extrasystoles. Cervical Doppler ultrasonography showed no significant abnormality. Cerebral computed tomographic scan demonstrated moderate atrophy with a chronic thalamic lacunae. Transesophageal echocardiography showed a partially mobile right ventricular mass, $1.5 \times 2.0 \mathrm{~cm}$, attached to the lateral wall just below the lateral tricuspid leaflet (Figure 1). No lesion was detected in the right atrium or the inferior vena cava, and the foramen ovale was not patent on ultrasound. Bilateral ventricular function was within normal limits with no tricuspid insufficiency. Computed tomographic scan of the thorax demonstrated a right ventricular mass, $2 \mathrm{~cm}$ in diameter, with thickening of the lateral ventricular wall but no extension from the inferior vena cava (Figure 2). Abdominal computed tomographic scanning showed a cystic lesion on the right kidney. The preoperative coronary angiogram showed no abnor-

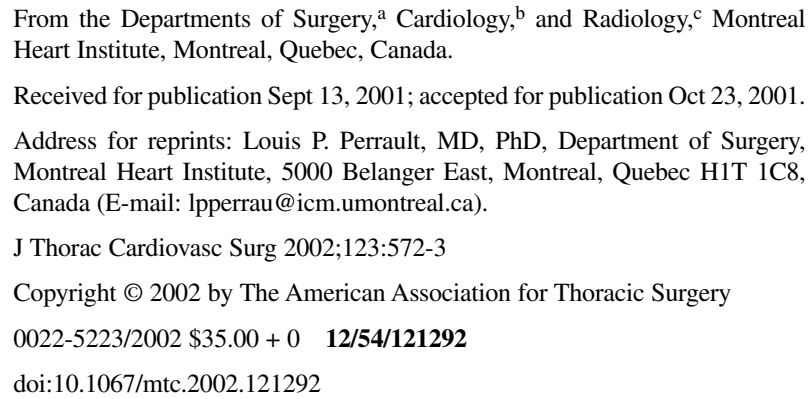

malities. Surgical treatment was undertaken to obtain a diagnosis and for a possible resection.

After median sternotomy and bicaval cannulation, intraoperative exploration revealed a rubbery mass at the acute margin of the right side of the heart close to the interventricular septum. Cardiopulmonary bypass was instituted with antegrade cardioplegia. A right atriotomy was performed and, on application of upward pressure on the right ventricle, a $2-\mathrm{cm}$ red, highly vascularized mass with a 5-mm pedicle was expulsed from the septal border. Two other lesions of 8 and $5 \mathrm{~mm}$ were discovered more medially in the right atrium. Excisional biopsy of the main pediculated mass and satellite lesions was performed. The thickened myocardium involving the right half of the ventricle was not resected. On irrigation of the right atrial cavity to test the competence of the tricuspid valve, a 4-mm wide atrial septal defect was identified and subsequently closed with a polypropylene 4-0 running suture. Postoperative transesophageal echocardiography showed no abnormalities. The postoperative course was uneventful. Pathologic examination of the lesions confirmed the diagnosis of cardiac metastases from renal cell carcinoma with right ventricular invasion. The patient was referred to the medical oncology department for systemic therapy. At 6 months' follow-up, there were no recurrences of transient ischemic attacks or evidence of distant spread.

\section{Comment}

Almost every type of malignant tumor has been shown to metastasize to the heart. Secondary cardiac neoplasias are 20 to 40 times more common than primary tumors. ${ }^{3}$ Leukemia is the most frequent cause of cardiac tumors, with cardiac lesions developing in $50 \%$ of patients with leukemia. Other neoplasias that produce cardiac metastases include breast, lung, melanoma, and lymphoma. Abdominal and pelvic tumors can also grow in a cephalad direction via the inferior vena cava to reach the right atrium ${ }^{3}$ at the time of presentation of the primary tumor. Such tumors most frequently include renal cell carcinoma, but extension from hepatic, adrenal, and uterine neoplasias has been reported. Ten percent of renal cell carcinomas involve the inferior vena cava and $40 \%$ of these extend into the right atrium. ${ }^{3}$ Surgical therapy for these patients typically requires cardiopulmonary bypass and circulatory arrest, with a 5year survival of $75 \% .^{3}$

This case report describes the occurrence of a blood-borne cardiac metastasis from a renal cell carcinoma. Many groups have demonstrated tumor thrombus extending through the inferior vena cava into the right cardiac cavities, ${ }^{2,4}$ but the occurrence of a distant metastasis to the heart from a hypernephroma years after pri- 


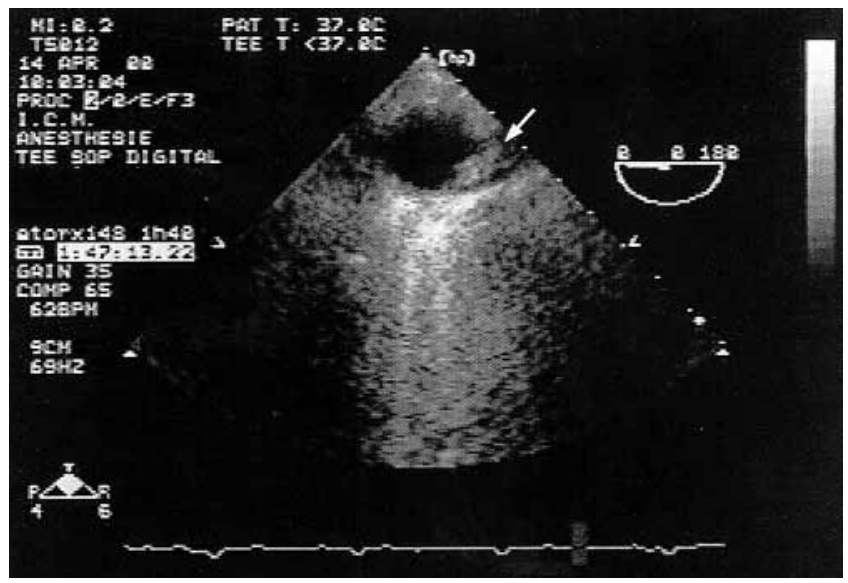

Figure 1. Transesophageal echocardiogram showing a partially mobile right ventricular mass, $1.5 \times 2.0 \mathrm{~cm}$, attached to the lateral wall just below the lateral tricuspid leaflet.

mary resection and its presentation as a transient ischemic attack by embolization through a patent atrial septal defect has never been reported. Excisional biopsies of the lesions responsible for the transient ischemic attack were performed with cardiopulmonary bypass. Complete resection of the tumor infiltrating half of the right ventricular myocardium was not undertaken.

\section{References}

1. Croft LB, Goldman ME. Images in clinical medicine: echocardiographic detection of a renal-cell carcinoma. $N$ Engl J Med. 1998;338:1279.

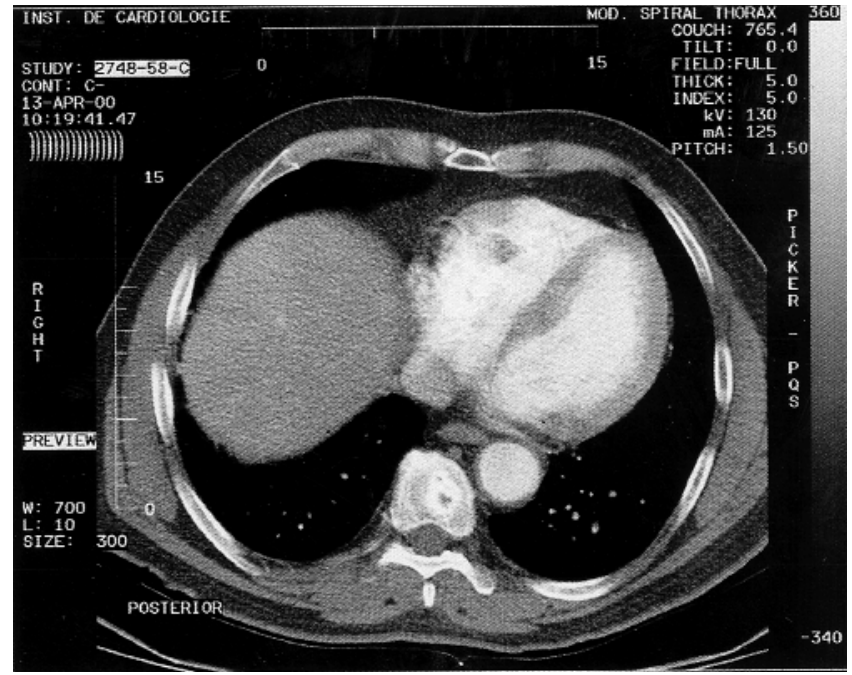

Figure 2. Computed tomographic scan of the thorax demonstrating a right ventricular mass $2 \mathrm{~cm}$ in diameter with thickening of the lateral ventricular wall but no extension from the inferior vena cava.

2. Chatterjee T, Muller MF, Carrel T, Kaufmann U, Meier B. Renal cell carcinoma with tumor thrombus extending through the inferior vena cava into the right cardiac cavities. Circulation. 1997;96:2729-30.

3. Edmunds LH Jr. Cardiac neoplasms. In: Cardiac surgery in the adult. McGraw-Hill; 1997. p. 1345-62.

4. Fanous H, Faddoul A, Constantinople NL, Spence IJ. Renal cell carcinoma extending into vena cava and right atrium. Urology. $1983 ; 22: 215-8$. 